\title{
Hepatic biliary lipid secretion and gall bladder biliary lipid mass in gall stone patients: effect of ursodeoxycholic acid
}

\author{
A Lanzini, T C Northfield
}

\begin{abstract}
We have carried out overnight measurements of hepatic secretion rate and duodenal output of biliary lipids using a duodenal perfusion technique. We correlated these measurements with the fasting state mass of biliary lipids within the gall bladder on the following morning using a combined nasoduodenal intubation and isotope scanning technique. We studied six gall stone subjects before and during treatment with ursodeoxycholic acid $675 \mathrm{mg} /$ day. Lipid mass within the gall bladder correlated with the corresponding overnight hepatic secretion rate for all three biliary lipids. During ursodeoxycholic acid treatment, there was an increase in gall bladder bile acid mass without significant change in cholesterol or phospholipid mass. We conclude that the mass of individual biliary lipids within the fasting gall bladder is influenced by overnight hepatic biliary lipid secretion rate; and that the effect of ursodeoxycholic acid $(675 \mathrm{mg} /$ day) on cholesterol saturation index of fasting gall bladder bile is mediated via an increase in bile acid mass rather than through a decrease in cholesterol mass within the gall bladder.
\end{abstract}

Oral administration of ursodeoxycholic acid reduces cholesterol saturation index of fasting gall bladder bile and dissolves gall stones in selected patients. ${ }^{12}$ It is not known whether the effect on cholesterol saturation index is caused by an increase in the absolute mass of bile acid and phospholipid within the gall bladder, or a decrease in cholesterol mass, or a combination of these effects. Only indirect information is available, based on measurements of bile acid pool size and duodenal output of biliary lipids. ${ }^{34}$ Biliary lipid output measurements have given conflicting results. Although Nilsell $e t a l^{3}$ and Von Bergmann et $\mathrm{al}^{4}$ are in agreement in reporting a decrease in duodenal cholesterol output, Nilsell $e t a l^{3}$ found bile acid and phospholipid output to be unchanged, and Von Bergmann et $a l^{4}$ reported an increased output for both bile acid and phospholipid during ursodeoxycholic acid administration. Both groups reported an increase in total bile acid pool size.

The aim of our study was to clarify the mechanisms involved by measuring the absolute mass of bile acid, cholesterol, and phospholipid within the fasting gall bladder before and during ursodeoxycholic acid treatment. We also studied factors affecting biliary lipid mass by measuring overnight hepatic secretion rates and duodenal output for bile acid, cholesterol, and phospholipid. Thus, we were able to explain the effect of ursodeoxycholic acid in terms of hepatic secretion ('the input') and duodenal output ('the output'), and in terms of lipid mass in the gall bladder ('the resultant').

\section{Methods}

SUBJECTS

We studied six subjects (three men and three women, age range 49-70 years, mean (SD) of $61 \cdot 3(8.4)$ years; body weight range $48-90 \mathrm{~kg}$, mean (SD) of $66 \cdot 2(14 \cdot 1) \mathrm{kg}$ ) with radiolucent gall stones in radiologically functioning gall bladders, after obtaining written informed consent. Radiation exposure was calculated to be $<0.05 \mathrm{rad}$ for the gut and the gall bladder wall in all subjects. The study was approved by the Local Hospital Ethical Committee in September 1981 .

\section{EXPERIMENTAL DESIGN}

All subjects were studied on and off ursodeoxycholic acid treatment. Four subjects (nos 1-4 in Table I) were allocated to receive baseline studies first, and two (nos 5 and 6 in Table I) to receive during treatment studies first. After completion of the baseline studies, the first group was started on treatment and repeat studies were carried out six to eight weeks later. The second group was studied six weeks after starting treatment, and had repeat studies six weeks after stopping it. Treatment consisted of ursodeoxycholic acid (Gipharmex, Milan, Italy) $675 \mathrm{mg} /$ day as a single bedtime dose. When studied during the treatment regimen, the subjects received the ursodeoxycholic acid dose five hours after the evening meal. The dose on a body weight basis ranged from $7 \cdot 5$ to $14 \cdot 1 \mathrm{mg} / \mathrm{kg} /$ day (mean (SD): $10 \cdot 6(2 \cdot 2) \mathrm{mg} / \mathrm{kg} /$ day $)$.

\section{CLINICAL PROCEDURE}

These were as described previously. ${ }^{56}$ On the day of the study, the fasting subjects were admitted to a clinical investigation ward. A four

TABLE I Clinical data

\begin{tabular}{lllll}
\hline & Age $(y r)$ & Sex & $\begin{array}{l}\text { Body weight } \\
(\mathrm{kg})\end{array}$ & $\begin{array}{l}\text { UDCA dose } \\
(\mathrm{mg} / \mathrm{kg} / \text { day })\end{array}$ \\
\hline 1$)$ & 54 & $\mathrm{~F}$ & 60 & $11 \cdot 3$ \\
$2)$ & 70 & $\mathrm{~F}$ & 48 & $14 \cdot 1$ \\
$3)$ & 69 & $\mathrm{M}$ & 61 & $11 \cdot 1$ \\
$4)$ & 61 & $\mathrm{M}$ & 90 & $7 \cdot 5$ \\
$5)$ & 65 & $\mathrm{M}$ & 71 & $9 \cdot 5$ \\
$6)$ & 49 & $\mathrm{~F}$ & 67 & $10 \cdot 1$ \\
\hline
\end{tabular}

UDCA = ursodeoxycholic acid. 
lumen nasojejunal tube was advanced under fluoroscopic control to position the proximal outlet in the body of the stomach, and the duodenal perfusion outlet opposite the ampulla of Vater. The duodenal aspiration tube was positioned at the ligament of Treitz, $20 \mathrm{~cm}$ beyond the perfusion tube (mixing segment). A more distal reinfusion tube was placed approximately $30 \mathrm{~cm}$ beyond the ligament of Treitz. The position of the tube was checked in the afternoon before the start of the duodenal perfusion study and the following morning at the end of the study.

Three equicaloric meals ( $500 \mathrm{kcal}$ each) were administered through the gastric tube at $9 \mathrm{am}, 12$ noon, and $630 \mathrm{pm}$, each meal being taken within a period of 10 minutes. The meals consisted of amino acids, $55 \mathrm{~g}$ (casein hydrolysate, Scientific Hospital Supplies, UK) and glucose polymer, $75 \mathrm{~g}$ (Caloreen, Roussel, UK) in $230 \mathrm{ml}$ tap water $\left(37^{\circ} \mathrm{C}\right)$. The meal did not contain any cholesterol or phospholipid.

\section{Duodenal biliary lipid output}

Hourly outputs of biliary lipids into the duodenum were measured using the duodenal perfusion technique of Northfield and Hofmann. ${ }^{7}$ A non-absorbable duodenal recovery marker (polyethylene glycol) was used for measurement of bile acid output into the duodenum. For measurement of cholesterol and phospholipid duodenal output and absorption, absorbable recovery markers $\left({ }^{3} \mathrm{H}\right.$-cholesterol and ${ }^{14} \mathrm{C}$ lecithin) were used. Polyethylene glycol (molecular weight range $3300-4000,12.5 \mathrm{~g}$ ) was dissolved in $51 \mathrm{NaCl} 154 \mathrm{mmol} / \mathrm{l}$ solution. 50 $\mu \mathrm{Ci} \quad{ }^{3} \mathrm{H}$-cholesterol (1,2 (n)-- ${ }^{3} \mathrm{H}$-cholesterol, Amersham International, UK) and $10 \mu \mathrm{Ci}{ }^{14} \mathrm{C}$ lecithin (L-3-phosphatidyl n-methyl- ${ }^{-14} \mathrm{C}$-choline dipalmitoyl, Amersham International, UK) were dissolved in $2 \mathrm{ml}$ diethyl ether together with non-radioactive carriers (cholesterol $2 \mathrm{mg}$ and lecithin $5 \mathrm{mg}$ ) and added to $10 \mathrm{ml}$ water. The ether was evaporated under a stream of nitrogen and the remaining mixture was sonicated (model 1510, Braunsonic, UK) for 20 minutes at 300 watt into an optically clear dispersion and added to the polyethylene glycol solution. The perfusate was maintained at $37^{\circ} \mathrm{C}$ in a water bath, magnetically stirred and perfused intraduodenally at a constant rate $(5 \mathrm{ml} / \mathrm{min})$ by a peristaltic pump (model 501, Watson Marlow, UK). The duodenal perfusion started at $5 \mathrm{pm}$ and, after allowing 60 minutes to achieve a steady state, duodenal content was aspirated by siphonage at hourly intervals for the next 12 hours. An aliquot of the aspirate (consistently $<5 \%$ of the total) was retained for analysis, and the remainder reinfused into the intestine through the distal tube to maintain the enterohepatic circulation of bile acids.

\section{Hepatic biliary lipid secretion}

This was measured by relating duodenal outputs to the duodenal recovery of indocyanine green which was continuously infused intravenously (iv) during the whole study, ${ }^{8}$ as described previously. ${ }^{56}$ By the time duodenal aspiration was started at $6 \mathrm{pm}$, the indocyanine green iv infusion had been given for the three hour period needed to equilibrate its iv infusion rate with its hepatic output. ${ }^{8}$

\section{Biliary lipid mass within the gall bladder}

This was measured as previously described ${ }^{59}$ at the end of the duodenal perfusion study. In brief, $5 \mathrm{mCi}{ }^{99 \mathrm{~m}} \mathrm{Tc}$ HIDA sodium $\mathrm{n}-\mathrm{n}(2,6-$ diethylphenlyl-carbamylmethyl iminodiacetate, Amersham plc, Bucks, UK), was injected iv at $3 \mathrm{pm}$. On the following morning the residual ${ }^{99 m}$ Tc HIDA activity in the gall bladder was measured by anterior and posterior abdominal scanning using a gamma camera/computer system (model Sigma 4105/MCS-560, Technicare, USA). The reason for giving ${ }^{99 m} \mathrm{Tc}$ HIDA the day before the gamma camera study was to enable us to measure its duodenal output during the whole study for simultaneous measurement of absolute gall bladder emptying and filling as reported in a separate paper. ${ }^{5}$ Data obtained from abdominal gamma camera counting were stored in the computer and later reanalysed using an area of interest facility to outline the gall bladder area as described previously. ${ }^{5}$ A sample of gall bladder bile was collected after the abdominal gamma camera study by stimulating gall bladder contraction with intravenous cholecystokinin (Boots, UK) for measurement of biliary lipid mass as described previously. ${ }^{59}$

\section{LABORATORY METHODS}

Polyethylene glycol concentration was measured in the perfusate and in duodenal aspirate by the turbidometric method of Hyden. ${ }^{10}{ }^{14} \mathrm{C}$ and ${ }^{3} \mathrm{H}$ activity was measured in the perfusate and in duodenal aspirate by liquid scintillation spectroscopy. ${ }^{6}$ Bile acid, ${ }^{11}$ cholesterol, ${ }^{12}$ and phospholipid ${ }^{13}$ concentrations in duodenal aspirate and in gall bladder bile samples were measured enzymatically. Within 24 hours from collection, indocyanine green concentration was measured spectrophotometrically as previously described. ${ }^{5}$

During ursodeoxycholic acid studies, duodenal aspirate contained both endogenous conjugated bile acid and orally administered unconjugated ursodeoxycholic acid. The two classes of bile acids were separated by ion exchange column chromatography. ${ }^{614}$ We based our calculations of duodenal output and hepatic secretion rate on the values obtained for conjugated bile acid alone.

\section{CALCULATIONS AND EXPRESSION OF} RESULTS

Cholesterol saturation index of fasting gall bladder bile was calculated using the polynomial equation of Thomas and Hofmann, ${ }^{15}$ based on cholesterol solubility lines described by Hegardt and Dam. ${ }^{16}$ Results were not corrected for the percentage glyco-ursodeoxycholic acid. ${ }^{17}$ 
The formulae for calculating results were as follows:

(1) Bile acid (BA) and indocyanine green (ICG) duodenal outputs $=$ BA (or ICG) $\times$

$$
\frac{\mathrm{PEG}_{\mathrm{P}}}{\mathrm{PEG}_{\mathrm{a}}} \times \text { vol }_{\mathrm{p}} \times \text { time }
$$

(2) Cholesterol and phospholipid duodenal output $=$ Cholesterol $($ or phospholipd $) \times$

$$
\frac{\mathrm{DPM}_{\mathrm{p}}}{\mathrm{DPM}_{\mathrm{a}}} \times \text { vol }_{\mathrm{p}} \times \text { time }
$$

(3) Bile acid (BA), cholesterol (chol), and phospholipid (phos) hepatic secretion $=\mathrm{BA}$ (or chol or phos) duodenal output $X$

$$
\text { ICG iv input }
$$

\section{ICG duodenal output}

(4) Storage fraction for biliary lipids=hepatic secretion rate-duodenal output

(5) Gall bladder (GB) bile acid, cholesterol or phospholipd mass $=$ BA (or chol or phos) mass ( $1 \mathrm{ml} \mathrm{GB}$ bile $) \times$

TcHIDA activity in the GB

TcHIDA counts ( $1 \mathrm{ml} \mathrm{GB}$ bile)

where $\mathrm{BA}=$ conjugated bile acid concentration, chol=cholesterol concentration, phos $=$ phospholipid concentration, ICG=indocyanine green concentration, $\mathrm{PEG}=$ polyethylene glycol concentration, $\mathrm{DPM}=$ disintegration per minute, TcHIDA $={ }^{99 \mathrm{~m}}$ Tc HIDA, $\mathrm{a}=$ duodenal aspirate, $\mathrm{p}=$ duodenal perfusate, $\mathrm{vol}=$ volume .

\section{STATISTICAL ANALYSIS}

Results have been expressed as mean (SD). Statistical significance has been tested using the Student's $t$ test for paired observations. Parameters of regression lines have been calculated using the least square method.

\section{Results}

RELATIONSHIP BETWEEN GALL BLADDER MASS, DUODENAL OUTPUT, AND HEPATIC SECRETION RATE FOR BILIARY LIPIDS (Fig 1)

In order to extend the range of the measurements, we have combined the results obtained during the control period and during ursodeoxycholic acid administration. There was a significant correlation between 12 hour hepatic secretion and gall bladder mass for bile acid $(\mathrm{r}=0.64, \mathrm{p}<0.05)$ and cholesterol $(\mathrm{r}=0.59$, $\mathrm{p}<0.05$ ), and a similar trend for phospholipid $(\mathrm{r}=0.50, \mathrm{~ns})$. By contrast, duodenal outputs were not correlated with the corresponding gall bladder mass for any of the three biliary lipids $(\mathrm{r}=0 \cdot 15$, ns, for bile acid; $\mathrm{r}=0 \cdot 11$, ns, for cholesterol; and $\mathrm{r}=0 \cdot 10$, ns, for phospholipid). Storage fraction correlated with the corresponding gall bladder mass for bile acid $(r=0.89$, $\mathrm{p}<0.001)$, cholesterol $(\mathrm{r}=0.77, \mathrm{p}<0.005)$ and phospholipid $(r=0.68, \mathrm{p}<0.02)$.

RELATIONSHIP BETWEEN GALL BLADDER BILIARY LIPID MASS AND CHOLESTEROL

SATURATION INDEX

There was no relationship between cholesterol saturation index and either bile acid $(r=-0.33$, $\mathrm{ns})$, cholesterol ( $\mathrm{r}=0 \cdot 34$, ns), or phospholipid $(\mathrm{r}=-0.31, \mathrm{~ns})$ mass of fasting gall bladder bile in combined results obtained before and during ursodeoxycholic acid administration in the six subjects.

\section{EFFECT OF URSODEOXYCHOLIC ACID} (Fig 2 and Table II)

There was a large variation between individual subjects before treatment for biliary lipid mass in the gall bladder and for hepatic secretion rates. Despite these variations in baseline measurements, and despite the differences in the daily ursodeoxycholic acid dose/kg body weight administered to individual subjects, changes observed during ursodeoxycholic acid treatment were consistent in individual subjects. Cholesterol saturation index of fasting gall bladder bile was reduced in each individual during ursodeoxycholic acid administration, and the mean value fell from $1.68(0.55)$ to 0.93 $(0.29)(p<0.02)$. Gall bladder bile acid mass increased in five subjects, the mean value increasing from $3.8(1.8)$ to $6.3(3.2) \mathrm{mmol}$ $(\mathrm{p}<0.02)$ during ursodeoxycholic acid administration. Gall bladder phospholipid mass increased in four subjects, but the mean value did not change significantly $(1 \cdot 5(1 \cdot 0) v 2 \cdot 3(1 \cdot 3)$ $\mathrm{mmol}, \mathrm{ns})$. Mean gall bladder cholesterol mass was unchanged $(0.7(0.4) v 0.7(0.5) \mathrm{mmol}, \mathrm{ns})$.

Hepatic bile acid secretion rate increased from $10 \cdot 1(4 \cdot 7)$ to $13 \cdot 4(5 \cdot 3) \mathrm{mmol} / 12 \mathrm{~h}(\mathrm{p}<0 \cdot 02)$, and cholesterol secretion decreased from $1.4(0.4)$ to $1.0(0.5) \mathrm{mmol} / 12 \mathrm{~h}(\mathrm{p}<0.005)$ during ursodeoxycholic acid administration. Hepatic phospholipid secretion remained unchanged $(4.0$ $(1 \cdot 7)$ v $4.4(2 \cdot 1) \mathrm{mmol} / 12 \mathrm{~h}, \mathrm{~ns})$. The proportion of hourly samples having saturation index $>1$ was reduced from $73 \%$ to $12 \%$ during urso-
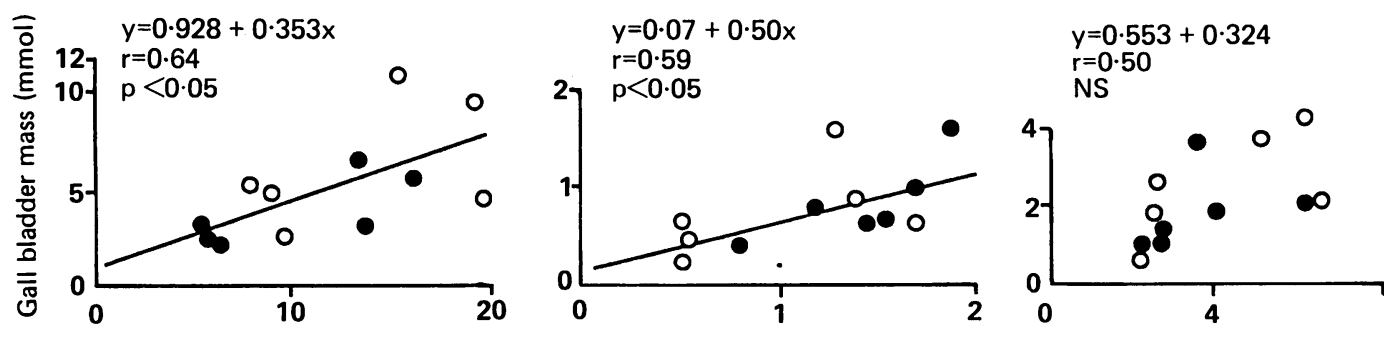

Hepatic secretion rate $(\mathrm{mmol} / 12 \mathrm{~h}$

Figure 1: Relationship between fasting state gall bladder mass and total 12 hour hepatic secretion for the three biliary lipids. Results are shown for all six subjects before ()$^{\prime}$ ) and during $(\bigcirc)$ ursodeoxycholic acid. 
Figure 2: Effect of ursodeoxycholic acid on fasting-state cholesterol saturation index (saturation index, criteria of Hegardt and Dam) and mass for all three biliary lipids within the gall bladder $(B=$ before ursodeoxycholic acid, $D=$ during ursodeoxycholic acid).

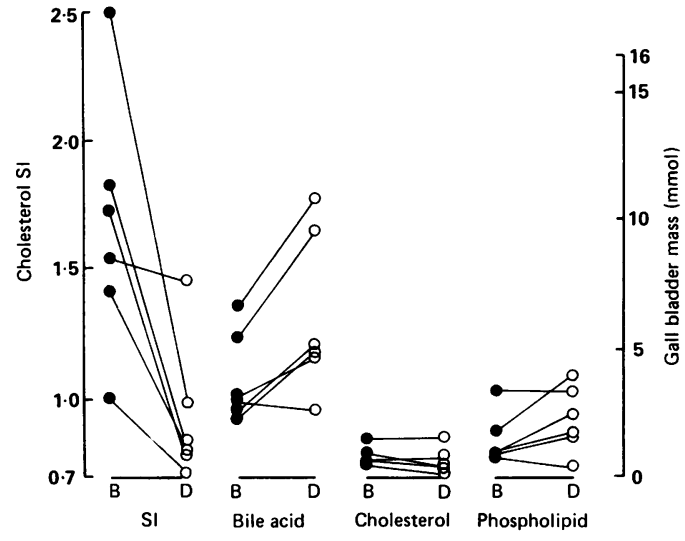

deoxycholic acid administration. There was no significant change in duodenal output for bile acid $(4.4(2.3)$ v $6.4(3.5) \mathrm{mmol} / 12 \mathrm{~h}$, ns), cholesterol $(0.6(0.2) v 0.4(0.3) \mathrm{mmol} / 12 \mathrm{~h}, \mathrm{~ns})$, or phospholipid $(1 \cdot 7(0.8) v 2 \cdot 0(1 \cdot 1) \mathrm{mmol} / 12 \mathrm{~h}$, ns) during ursodeoxycholic acid administration.

\section{Discussion}

We have previously shown good agreement between the indirect isotope scanning and intubation technique for measuring biliary lipid mass in the gall bladder and direct measurements on intact gall bladder contents after surgical removal of the gall bladder. ${ }^{18}$ Our duodenal perfusion technique has also been previously validated. ${ }^{5}$

We have now shown that biliary lipid mass in fasting state gall bladder bile is related to hepatic secretion rate of biliary lipids. This relationship is based on results obtained before and during ursodeoxycholic acid treatment, making it possible to expand the range of observations available, particularly for bile acid gall bladder mass (two-fold increase in range values, Table II). The fact that both sets of results are equally distributed along the single regression line further indicates that this relationship is physiological, at least in a qualitative sense. The relationship was clearly shown for bile acid and cholesterol. Although visual inspection of the individual data points indicates a trend for a positive correlation between hepatic secretion rate and gall bladder mass for phospholipid (Fig 1c), this was not statistically significant. These results are in keeping with our previous study on gall bladder motor function, showing that the major proportion of hepatic bile (at least $80 \%$ ) enters the gall bladder during both the postprandial and fasting period.

We have previously found a significant relationship between gall bladder cholesterol mass and saturation index in a homogeneous group of 10 young male non-obese healthy volunteers. ${ }^{9}$ We failed to find a relationship in the six gall stone subjects studied here. This discrepancy may be partly explained by the observation that, by contrast with our previous study on healthy volunteers, this group of subjects was not homogeneous in relation to sex, body weight, and age. These factors are all known to influence cholesterol secretion, and they may have also influenced cholesterol mass in the gall bladder to a different extent in different subjects.

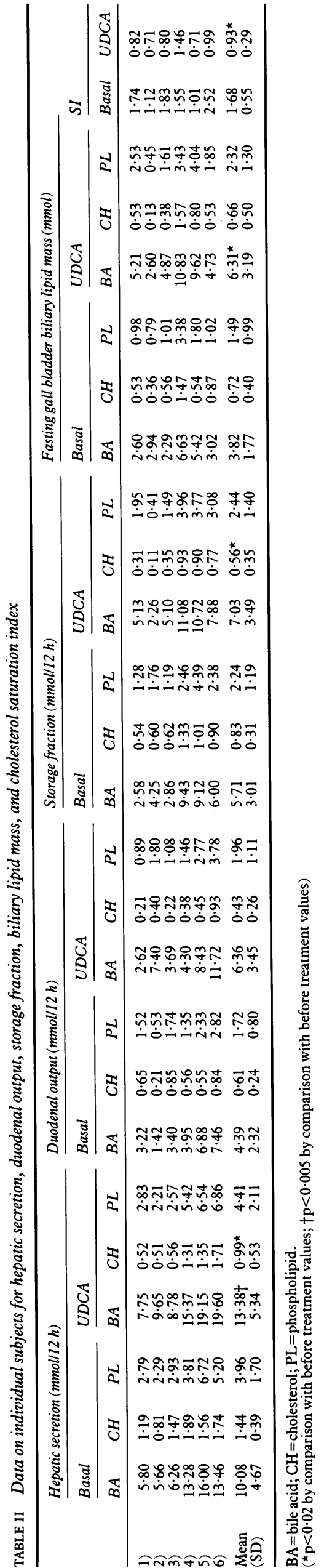


The magnitude of the effect of ursodeoxycholic acid on gall bladder bile acid mass (an increase of $2.5 \mathrm{mmol}$ ) is greater than that which can be attributed to the bedtime dose of ursodeoxycholic acid (approximately $1 \mathrm{mmol}$ ). Expansion of the bile acid pool has been reported by Nilsell $e t a l^{3}$ and by Von Bergmann $e t a l^{+}$after chronic administration of a similar dose of ursodeoxycholic acid, and this may explain the size of the increase in bile acid mass observed during treatment. A significant correlation has been previously reported between bile acid pool size and bile acid mass within the gall bladder in ten healthy male volunteers. ${ }^{9}$ The increase in bile acid mass during ursodeoxycholic acid treatment was accompanied by a non-significant trend for phospholipid mass. This increase in phospholipid mass occurred in only four subjects (mean increase $53 \%$ compared with $66 \%$ for bile acid). By contrast, there was absolutely no change in cholesterol mass (Fig 2). This latter finding could not have been predicted on the basis of biliary lipid secretion measurements, which have consistently shown a reduction in cholesterol secretion during ursodeoxycholic acid treatment. ${ }^{3+}$

An advantage of the technique used in this study was that it enabled us to distinguish the mechanism involved in the reduced cholesterol saturation index in hepatic bile from that involved in gall bladder bile during ursodeoxycholic acid administration. That the two mechanisms are different is clearly shown by this study. Our results for ursodeoxycholic acid indicate that when this is given at bedtime, the reduction in cholesterol saturation index of hepatic bile involves an increase in bile acid secretion rate together with a decrease in cholesterol secretion rate (Table I). By contrast, the reduction in cholesterol saturation index of fasting gall bladder bile involves an increase in bile acid mass in the gall bladder and no reduction in cholesterol mass (Fig 2). Although there is an increase in bile acid for both hepatic secretion rate and gall bladder mass, the relative increase was greater for the latter (approximately 85\%) than for the former (approximately 35\%), implying increased diversion of hepatic bile into the gall bladder. This increased diversion provides an explanation for the fact that the decrease in cholesterol secretion rate was not accompanied by a decrease in cholesterol mass in the gall bladder.

The fact that ursodeoxycholic acid treatment increased gall bladder storage of biliary lipids is consistent with our finding that hepatic bile acid secretion rate ('the input') increased during treatment without significant increase in duodenal bile acid output ('the output'). This may be explained by the observation of Forgacs $\mathrm{et} \mathrm{al}^{19}$ that gall bladder emptying is decreased in gallstone patients receiving ursodeoxycholic acid, consistent with our finding of an enhancement of gall bladder storage ('the resultant').

The authors wish to thank Gipharmex, Milano, Italy for financial support, and Miss Marion Amos for typing the manuscript. The present address of Dr A Lanzini is Istituto di Clinica Medica, Spedali Civili, 25100 Brescia, Italy.

1 Makino I, Shinozaki K, Yoshino K, Nakagawa B. Dissolution of cholesterol gallstones by ursodeoxycholic acid. $\mathcal{F} p n \mathcal{F}$ Gastroenterol 1975; 72: 690-702.

2 Fedorowski T, Salen G, Colalillo A, Tint GB, Mosbach EH, Hall JC. Metabolism of ursodeoxycholic acid in man. Gastroenterology 1978; 73: 1131-7.

3 Nilsell K, Angelin B, Leijd B, Einarsson K. Comparative effects of ursodeoxycholic and chenodeoxycholic acid on bile acid kinetics and biliary lipid secretion in humans. Gastroenterology 1983; 85: 1248-56.

4 Von Bergmann K, Epple-Gustfeld M, Leiss O. Differences in the effects of chenodeoxycholic and ursodeoxycholic acid on biliary lipid secretion and bile acid synthesis in patients with gallstones. Gastroenterology 1984; 87: 136-43.

5 Lanzini A, Jazrawi RP, Northfield TC. Simultaneous quantitative measurements of hepatic secretion and of absolute tative measurements of hepatic secretion and of absolute
gallbladder storage and emptying during fasting and eating in man. Gastroenterologv 1987; 92: 852-61.

6 Lanzini A, Northfield TC. Effect of ursodeoxycholic acid on biliary lipid coupling and on cholesterol absorption during
and eating and fasting in subjects with cholesterol gallstones. Gastroenterology 1988; 95: 408-16.

7 Northfield TC, Hofmann AF. Biliary lipid output during three meals and an overnight fast. Relationship to bile acid pool size and cholesterol saturation of bile in gallstone and control subjects. Gut 1975; 16: 1-11.

8 Van Berge Henegouwen GP, Hofmann AF. Nocturnal gallbladder storage and emptying in gallstone patients and healthy subjects. Gastroenterology 1978; 75: 879-85.

9 Jazrawi RP, Kupfer RM, Bridges C, Northfield TC. Assessment of gallbladder storage function in man. Clin Sci 1983; 65: 158-91.

10 Hyden S. A turbidometric method for the determination of higher polyethylene glycols in biological materials. Kungl higher polyethylene glycols in biological mat
Lantbrukshogskolans Annaler 1955;22: 139-45.

11 Talalay P. Enzymatic analysis of steroid hormones. Methods Biochem Anal 1960; 8:119is

12 Roda A, Festi D, Sama C, et al. Enzymatic determination of cholesterol in bile. Chim Clin Acta 1975; 64: 337-41.

13 Qureshi NY, Murphy GM, Dowling RH. The enzymatic determination of total phospholipids in bile and bile rich duodenal aspirates. Chim Clin Acta 1980; 105: 407-10.

14 Hall CN, Lanzini A, Facchinetti D, Northfield TC. Application of simplified methods of bile acid analysis to gastrointestinal fluids. Clin Sci 1983; 65: 57

15 Thomas PJ, Hofmann AF. A simple calculation of the lithogenic index of bile expressing biliary lipid composition on rectangular coordinates. Gastroenterology 1973; 65: 698700 .

16 Hegardt FG, Dam H. The solubility of cholesterol in aqueous solutions of bile salts and lecithin. $Z$ Ernahrungswiss 1971; 10: $223-33$.

17 Carey MC. Critical tables for calculating the cholesterol saturation of native bile. $\mathcal{F}$ Lipid Res 1978; $945-55$.

18 Jazrawi RP, Brown C, Northfield TC. Measurement of biliary lipid mass within the gallbladder in health and in ileal Crohn's disease [Abstract]. Gut 1984; 25: A1141.

19 Forgacs IC, Maisey MN, Murphy GM, Dowling RH. Influence of gallstones and ursodeoxycholic acid therapy on gallbladder emptying. Gastroenterology 1984; 87: 299-307. 